\title{
Mathematical Modelling from the Instrumentation of Improper Integrals
}

\author{
Mateus-Nieves, Enrique1, Hernández Montañez Wilfaver* \\ 1 Externado university of Colombia 1; jeman124@gmail.com \\ 2 Externado university of Colombia 2; ww.hhh.mmm.hernandez@gmail.com \\ * Correspondence: jeman124@gmail.com; Tel.: +057 304790941 (corresponding authors 1)
}

\begin{abstract}
Background: There is little clarity in the application of content related to improper integrals in university students, due to the absence of meaning, which prevents them from making a connection with everyday problem situations. Methods: we designed a mathematical modelling proposal where a specific situation involving the instrumentation, use and application of this type of integrals is experimented and solved with a population of engineering students, who learn to use them. Results: The importance of using mathematical modelling as a didactic-dynamic resource is highlighted because it helps students to reach an understanding of real situations involving improper integrals in different contexts. Conclusions: Despite the numerous errors detected in the students, this strategy made it possible to demonstrate the development of advanced mathematical thinking skills in young people.
\end{abstract}

Keywords: Improper integral; Mathematical modelling; Mathematical instrumentation.

\section{Introduction}

In university teaching practice, difficulties are observed in students taking Calculus I and II courses. There is little clarity in applying content to the solution of problem situations, which shows a lack of connection with the environment and with problems of everyday life, particularly with topics related to the subject Calculus II, where one of the topics is the learning of improper integrals ${ }^{1}$. This concept is of great importance for students who are training professionally in both mathematics and engineering due to the number of applications it has; among them, calculation of probabilities to define functional rules, calculation of Fourier and Laplace transforms, physical calculations such as work and energy, to name but a few.

We seek to provide elements that help to solve the need to show and manipulate applications of mathematical content, in concrete problems specific to professional work, because we believe they help to overcome the lack of clarity that students have about the application of content related to integrals, the lack of connection with the environment and with problems of everyday life. To this end, we selected and applied ten specific engineering problem situations that involve the use of this type of integrals. For reasons of space, in this report we present one of them, chosen because it offers elements of analysis that help to enrich the teaching and learning processes of this mathematical object. This is a subject that is little addressed in the existing literature.

From this selection, we describe a mathematical modelling proposal where the teacher experiments and solves a selected situation, based on an adaptation of the mathematical modelling circle proposed by [1] visualising it as a research instrument and at the same time as a 
didactic strategy. The selected situation was analysed from two phases in which the research was developed; as a strategy, the use of a free technological tool was incorporated.

Among the results found, the importance of using mathematical modelling as a didacticdynamic resource, which helps students to achieve a deep understanding of real situations, relating them to problems that can be mathematised, from the instrumentation of improper integrals in situations, both intra and extra mathematical. This strategy provides students with the possibility of taking a position in relation to the objects studied. This made it possible to demonstrate the development of advanced mathematical thinking skills in these students.

\section{Background}

[2] faced with the imbalance between the conceptual and algorithmic dimensions of Calculus, seeks to understand what elements make it possible to establish a connection between the concepts of Integral Calculus and the algorithms that characterise them. [3] state that the formal teaching of the concept of the integral, as a limit without any context outside the algebraic, can be difficult to understand even for advanced students if it does not take into account a concrete context. [4] state that traditional calculus teaching fails to make students recognise, understand or apply the concept of the integral in contexts that have not been studied in class. [5] propose a conceptual approach to calculus through the use of technological resources (specifically simulations). Where the teaching of the integral is not limited to the calculation of the area demarcated by a curve, as is traditionally done, but the activities are carried out within contexts corresponding to concrete situations.

[6] carries out a didactic analysis of how three groups of university students learn to use the method of integration by parts (MIP). She identifies difficulties in the students in recognising the type of integral involved in the situation posed. Shows use of the IPM algorithm mechanically, without understanding it, through repetitive exercises. Emphasises that it is possible for students to acquire, understand and apply them solidly if they understand them, that is, if they realise the relationship that they maintain with the relational structure of the problems to which they are applied. [7] presents an extension to the 2016 research, with a study for the integral from three dimensions: epistemic, to address the historical genesis of the concept; cognitive, to consider the difficulties that students present when faced with problem situations involving the concept. And didactic, where he proposes problem situations that aim to enrich the field of social practices that are addressed with the concept. From this study, it indicates that the mathematical notions for the integral have a high level of abstraction, fundamental in the development of advanced mathematics and difficult for students to learn, therefore, a study that takes into account the logical conditions involved in the process of constitution of this mathematical object, contributes to a better understanding of it and its possible articulation.

[8] present a study carried out with three groups of university students learning Integral Calculus. They redesign the curricular structure of the Calculus II course, articulating the epistemic, cognitive and didactic dimensions for the various meanings identified for the integral, seeking that students make sense of what in their work they 
call "partial meanings for the integral". This makes it possible to show the integral as a problem-solving tool, where students reach a level of abstraction that is fundamental in the development of advanced mathematics. They identify the implicit perception of a culture in the teacher and in the student, where learning to say what the integral is and to represent it geometrically, without having an understanding of it, is enough to pass the subject (presence of a purely formal-mechanistic approach). [9] from a case study, show that the teaching of Infinitesimal Calculus begins in secondary education and is formalised in the first semesters of university, where the transition from elementary mathematical thinking (EMT) to advanced mathematical thinking (AMT) is expected. Given that in higher education, progressive mathematization implies the need to abstract, define, analyse and formalise; among the cognitive processes of the psychological component, in addition to abstracting, those of "representing, conceptualising, inducing and visualising" [9] (p. 69) stand out. In this way, the aim is for students to "understand" this type of concepts through the exercise of algorithms specific to the subject and its properties.

[10] presents a reflection on working with improper integrals where he highlights that traditional teaching does not lead students to acquire the ability to understand that there is a mathematical object called "integral", which in turn is unitary and systemic. That it is made up of various meanings (types of integral), and that these can be used in various situations of both an intra and extra mathematical nature. It indicates that traditional teaching (mechanistic approach), evaluates students in such a way that they only apply an algorithm, iteratively, without understanding what they are doing or the benefits of using this tool. This type of teaching suggests that the important thing is to master the procedures for solving exercises, or to memorise definitions and understand the proof of theorems (formal approach), leaving aside the usefulness and richness of this mathematical entity for problem solving.

\section{Theoretical framework}

This work is supported by two conceptual axes: instrumental genesis theory and mathematical modelling as tools that allow the student to understand and articulate the meanings for the improper integral.

\subsection{Instrumental Genesis Theory}

[11] propose this theory understood as the help of technological elements that the student can incorporate in the construction of mathematical thinking schemes through manipulation and understanding of guided computer elements that allow the generation of valid tools for the acquisition of mathematical competences that help him/her to solve problems related to his/her context. This theory outlines two fundamental forms of cultural behaviour: the use of tools and human language. This position places activities with tools at the centre of the problem of the constitution and functioning of man's higher psychic functions. It proposes the use of mediating instruments in the construction of higher mental processes with the use of technology, from two aspects: Artefact: referring to all objects of material culture to which the learner has access during his or her 
development. Instrument: psychological construct that is generated through the manipulation of the artefact. It proposes that to the extent that the relationship between artefact and user acquires meaning and over time its usefulness is known and internalised, it becomes an instrument. Hence, educational software can be seen at a certain point as an artefact, as it lacks contextualisation and adequate instructions for the student to determine its importance in the learning of a given subject. It is vital that, over time, the teacher uses precise strategies so that the student turns the educational software-artefact into an educational software-instrument in the solution of mathematical problems.

It is here where we articulate this work with mathematical modelling, given that any artefact can become a learning tool, regardless of whether it aims to generate a EMT or an AMT, which implies: precision with which it is explained, usefulness, operation and adaptability to the training intentionality. In terms of [12] (p. 96) "a knowledge content that has been designated as knowledge to be taught, undergoes a set of adaptive transformations that will make it suitable to occupy a place among the objects of teaching", a process known as didactic transposition. This implies that the teacher modifies his or her didactic structures and transforms an artefact, educational software, into a learning tool that allows the student to establish a constant relationship with this teaching object. In this respect, [11] state that:

The instrument does not exist in itself, a machine or a technical system does not immediately constitute a tool for the subject. Thus, an instrument results from the establishment, by the subject, of an instrumental relationship with the artefact, whether material or not, produced by others or by himself (p. 84).

[13] presents different relationships that occur under a model of situations of the instrumented activity, from the modelling of the subject-instrument-object triad. Initially, direct relationships are found in a respective manner: subject-instrument, instrumentobject and subject-object, characterising two techniques: instrumentalization, a process of enrichment of the properties of the artefact by the subject. Instrumentation, progressive discovery by subjects of the intrinsic properties of the artefact accompanied by schema accommodation and changes in the meaning of the instrument, resulting from the association of the artefact with new schemas. It refers to Instrumentation focused and directed towards the subject, towards the operative handling of the artefact; it indicates that it occurs from the instrumentalization stage onwards and is directed towards the cognitive, towards the subject's inner self and the way in which the subject acquires schemes of instrumented action in the solution of specific tasks.

\subsection{Mathematical modelling}

We adopt the theoretical position proposed by [1]. He presents it as the process of constructing a directed model of a real situation into a mathematical model, a way of connecting the real world with mathematics. He proposes the need to schedule a sequence of activities that he calls: modelling circle, composed of seven steps: construction, structure, mathematization, mathematical work (resolution), interpretation, 
validation and exposition. He indicates that in each step it is possible to highlight difficulties and progress in the students, as a way of improving the teaching and learning processes.

During the modelling circle, the student is expected to replace the cognitive object with its mathematical image, a mathematical model, combining theory with experiment, an event that is easier if we use technological tools such as the computer and different mathematical software. [10] (p. 201) state that "the process of constructing abstract concepts such as the integral is difficult for students to understand", therefore, in this work we assume mathematical modelling as a didactic tool to strengthen the teaching and learning processes of this type of integrals. To this end, the first two steps in the modelling cycle, construction, structure, were previously worked on with the sample, where the definite integral was presented and conceptualised from different meanings: anti-derivative of a function; area under the curve; notion of accumulation and rate of change as mutually dependent processes. The aim was for the students to interpret these different meanings, articulating them to different problem situations, initially to situations of a purely intra and later to other extra-mathematical situations. In the results section, one of these situations is shown in detail and some of the ten selected are mentioned.

\section{Methodology}

This is an action-research with a qualitative approach, where the intervention seeks to demonstrate changes in the target population, university students taking the subject calculus 2 at a university in Bogota. The sample, 50 students from three engineering degrees (Cadastral, Electrical, Mechanical), and two professors who teach the subject to these three groups. We sought to articulate instrumentation with mathematical modelling as a teaching methodology; we chose ten problem situations typical of engineering whose solution involves the use of this type of integrals. The work was carried out in two phases: 1) awareness-raising, 2) implementation and evaluation. We organised the sample into subgroups of three students to carry out the programmed activities, so that we could have an exhaustive follow-up of the development achieved by each student.

The awareness-raising phase was carried out over 8 weeks; the objectives set for the first two steps - construction and structure - were met, as shown in Table 1. This allowed both teachers and students to approach the process, which is here called mathematical pre-modelling, from the development of two strategies: 1) Presenting programme contents based on known mathematical models, e.g., the definite integral taught as a technique for calculating an anti-derivative (presentation and handling of the algorithm). Then as the area under the curve for various functions; these were first graphed in the notebooks, then using GeoGebra online and other freely available online graphing programs of the students' choice. We sought to familiarise both teachers and students with the problem situation; they were able to visualise the graph of the region, identify, relate and extend the meaning of the integral as a ratio of change, facilitating an approach to the notion of accumulation, as mutually dependent processes. 2) To implement in logicalnumerical algorithms (from the chosen software), the qualities present in the given 
situation. The objective for this phase was to identify, describe and analyse the actions that students carry out when facing problem situations, meaning ${ }^{2}$ assigned to a mathematical object, which require the use of improper integrals with functions of one variable.

The second phase of implementation sought to achieve the objectives outlined in the remaining five steps (see table 1), to complete the mathematical modelling circle, and then the experience was evaluated. The objective of this phase was to ensure that the students were able to mathematise the problem situation posed, interpret the possible ways to find a solution and validate whether the answer reached satisfies the needs set out in the situation. Subsequently, the teacher anonymously shared the results with the group, regardless of whether or not the answer found satisfied the requests of the problem; the idea of anonymity was to achieve security and confidence in the students.

In both phases, the work in subgroups sought to get the students to discuss, analyse, construct, investigate possible solutions to the proposed situations, and finally deliver a written report, where only the researchers knew to which subgroup each production corresponded. These reports were discussed in plenary with the general group, the interest of anonymity in this stage of evaluation was that no student would feel at a disadvantage with respect to their peers when their work was exposed. As a pedagogical strategy, the teacher presented each work to the plenary, submitted it to the analysis and observations of the general group, they had to check whether the modelling proposal offered met the needs set out in the problem, whether they were sufficient or incomplete.

\section{Results and discussion}

In order to articulate the instrumentation with the steps proposed in the mathematical modelling circle distributed in the two phases of the research, it was necessary to go through the instrumentalization. We articulated them because we start from the premise that the incorporation of technology as part of the process, deploys autonomy in the students with respect to the teacher; it allows them to develop skills for the search for information and greater participation and interest in the class. Table 1 illustrates the process followed. At the end of the semester, each subgroup submitted a written report as support for the work done, this time no longer anonymously, in which they made the necessary adjustments to the 10 situations proposed, in accordance with the plenary discussions on their suitability and the formalisation of the programme content by the teacher. 
Table 1. Articulation between instrumentation vs. steps of the mathematical modelling circle

\begin{tabular}{clc}
$\begin{array}{c}\text { Instrumental } \\
\text { Genesis }\end{array}$ & Work & $\begin{array}{c}\text { Steps of the } \\
\text { modelling circle }\end{array}$ \\
activities & & \\
\hline
\end{tabular}

Present the algorithm for calculating an anti-derivative (presentation and handling of the algorithm, Barrow's rule).

Construction Identify the relationship between the area under the curve and the defined integral.

Represent the function on the Cartesian plane and determine the region that defines the requested area.

requested area.
Use online software to identify and visualise the graph of the region.

Structuration Recognise that the definite integral of the rate of change of a quantity gives the net change of that quantity.

Associate that definite integrals: describe the accumulation of a quantity, so the definite integral gives the net change in that quantity.

Relate the rate of change and accumulation as distinctly dependent processes.

Indicate the area covered by the function in the assigned variation for $\mathrm{x}$.

Mathematization Recognise the area of the region as the definite integral in the given interval. (Riemann sum).

Identify, from the software, the volume and/or surface area of the solid of revolution.

Relate the volume and/or surface area as the result of calculating an improper integral.

Relate the surface area of the three-dimensional figure to the two-dimensional area previously

Resolution calculated.

Relate the units of measurement for each case.

Establish the solid of revolution that represents the given situation.

Calculate volume and/or surface area of the solid of revolution and express the result using

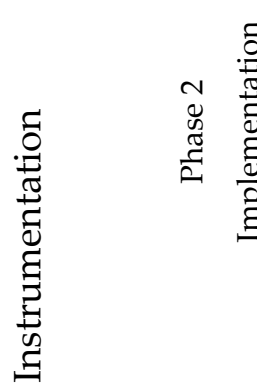
the specific units of measurement.

Express concretely and in real context the results found related to the surface area and volume of the solid generated.

Interpretation Recognise the units of measurement of the surface area and the volume generated by the solid. Express and record correctly in the software the intervals in which the function representing the proposed problem situation is analysed.

Diagram the corresponding solid in the software.

Construct three-dimensional figures from well-defined functions, digitised in the software of

Validation a solid of revolution.

Check whether the results obtained correspond to the situation posed and satisfy the requested needs.

Exposition Socialise the process developed throughout the experience.

Coherently support the work developed and the possible extension to other possible situations 


\subsection{For the awareness-raising phase}

For reasons of space, we will only show one of the ten situations proposed here, because it offers elements of analysis that we believe contribute to the research. During the approach to mathematical pre-modelling as a strategy, the students had already learned what a definite integral is and how it is calculated. We asked them to graph in their notebooks the function $f(x)=\frac{1}{x^{\prime}}$ to calculate the area of the region included in the interval $1 \leq x \leq 3$. They then had to explain how they did this process and what the value of the area see (see Figure 1a). Then they had to switch to the software and perform the calculations. Compare results and decide on the benefits of using the technology (Figure $1 b)$. Figures $1 \mathrm{a}$ and $1 \mathrm{~b}$ show the output of student E13.
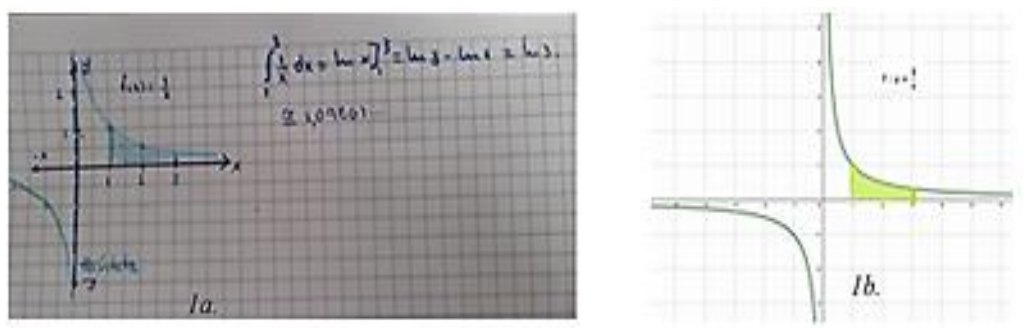

Figure 1. (1a) Production in student E13's notebook, (1b) production with student E13's software.

They were then asked to graph and interpret what will happen if we extend the interval of integration between $1 \leq x<\infty$, (see Figure 2a); we asked to express the integral. They were asked: if possible, does this integral allow them to calculate the area of the region bounded by the curve in the given interval? The answer obtained was not difficult for them to identify, they presented the expression $\int_{1}^{\infty} \frac{1}{x} d x$, despite the fact that until that moment it had not been defined that this register corresponds to a new integral, this time called improper, which implies additional work to that applied to the definite integrals. Some students mathematised using pencil, paper and calculator trying to find an answer, student E3 mentioned: "Professor is math error, did we do something wrong? The teacher in charge asked how they did the work? This group did not manage to answer when another classmate, E21 mentions: "we did not do it in the notebook, we used the software and it says that the integral is divergent, what does this mean, teacher?" (Figure 2b) E39 says: teacher, why does each subgroup have a different answer? In the activities given to the students, we asked them to extend the concept by rotating this figure about the $\mathrm{x}$-axis of the plane ${ }^{3}$, they were to plot the graph on paper, Figure 2c, shows the output of student E24. Then they had to repeat the process in the software, compare images, discuss the situation and think about whether it was possible to calculate the volume and surface area of that portion of solid that was formed, which in mathematics is called "Torricelli's trumpet" or "Gabriel's horn". 

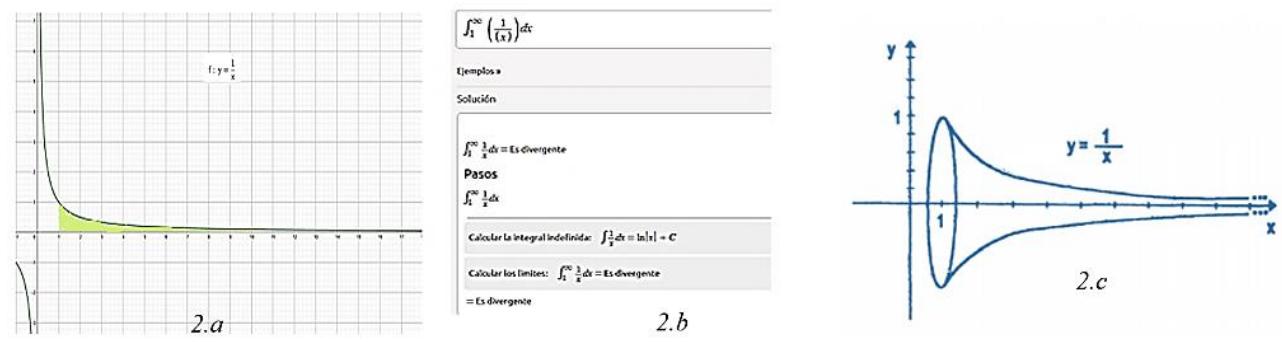

Figure 2. (2a) plot of the curve in the interval $1 \leq x \leq \infty$. (2b) student E21 output in the software, (2c) output of student E24

.The subgroup of student E19 using (GEOGEBRA applets online) provided the related images in Figure 3 to answer the above questions. This 3D visualization allowed them to observe variation in the size of the solid as $x$ approached infinity or moved back towards 1 , as well as the formation of the solid as $y$ varied.
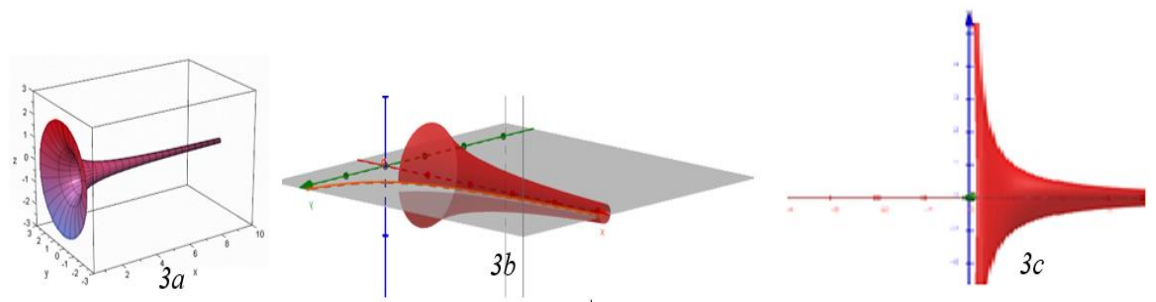

Figure 3. (3a) Gabriel's horn', (3b) Torricelli's trumpet ${ }^{5}$, (3c) Torricelli trumpet when $x \in(0, \infty)$ is analysed

.From this situation we can analyse: it is a problem framed within a purely mathematical context. It is about a function that is on an open interval on the right, for which we are asked to try to calculate the area (the integral) using graphical and numerical methods. We can see two types of students, those in the first group are more confident in their knowledge and take the risk of doing the work using their notebook and calculator, those in the other group use the software directly. Both types of students use graphical and algebraic semiotic representation registers. The graphs are well done by all of them, situated above the axis in the requested interval, then construction of the solid of revolution. This shows adequate handling of instrumental procedures, use of graphic and symbolic representations. But when calculating the area of the region, they expected approximate values of the total area as if it were a "definite" integral. Students using the software, when looking at the answer "the integral diverges" (Figure 2b), are puzzled because they expected a numerical figure. The students who did their work in the notebook make mistakes when they take the extremes of the integration interval at the points of discontinuity of the function, in this case $\infty$ and evaluate them in the same way as in Figure 1a. This error could be related to at least two central concepts of calculus: continuity and completeness of the real numbers ${ }^{6}$ not covered in this work.

We observe in the students that the discontinuity of the function leads them to make a series of mistakes where they apply the graphical, numerical and algebraic methods as routine procedures without questioning the best way to solve the problem. The algebraic resolution of the problem is extrapolated directly from the one applied to the definite 
integral, E18 mentions: "what we did was to apply Barrow's rule, then we tended the limit and it gave us a value". Students who used the software directly are not able to establish a connection between the results obtained with the algebraic and graphical registers, they are unable to coherently identify the information provided by the problem and they do not coherently coordinate the different registers used with the data provided by the software. They solve the task correctly, but do not know how to interpret it because they do not know the process followed (step by step), carried out by the software. It is worth noting that some students repeating the subject identified that it was an improper integral, instead of the ordinary Riemann integral, however, in the interpretation, they fail because they find it difficult to express in a concrete way and in a real context the results found related to the surface area and the volume of the solid generated. For these students it is still not clear that applying an improper integral where one of its limits is infinite is related to the evaluation of the integral of a probability density function?

Subsequently, the teacher in charge experimented, solved and formalized the existence of improper integrals from this specific situation, determining the necessary conditions to classify them as first, second or third species. To formalize third-species integrals, the question was posed: is it possible to consider Torricelli's trumpet when $x \in$ $(0, \infty)$ (Figure 3c), and from there this type of integral was defined as the sum: $\int_{0}^{\infty} \frac{1}{x} d x=\int_{0}^{a} \frac{1}{x} d x+\int_{a}^{\infty} \frac{1}{x} d x, a \in(0, \infty)$

Based on this pre-modelling, other situations were proposed in which the students had to sketch a possible mathematization that would allow them to find some kind of feasible solution. The sequence of programmed activities was designed in such a way that students could justify the processes followed. In order to establish concepts, we used analogous examples that could be related to their everyday life, e.g., calculating currents, capacitances, current charge and discharge times; determining the forces exerted by a fluid on the end of a tank, among others. We were able to verify that by this time the students and teachers were ready to tackle the necessary steps involved in the modelling circle methodology. Thus, during the remaining time of the semester, eight weeks, situations were developed in which it was possible to accompany the learning process and observe the strengths, difficulties and results of the students involved in this work.

\subsection{Implementation of the mathematical modelling stage}

For this phase we sought to articulate the five missing steps to complete the modelling circle (mathematization, resolution, interpretation, validation and exposition) responding to the objectives outlined in Table 1 . In this phase we took up the work advanced by the students when they constructed the graph on paper for the function $\int_{1}^{3} \frac{1}{x} d x$, then on $1 \leq x \leq \infty$. Subsequently it was formalized that, by rotating this figure about the $x$-axis of the plane, it generates a solid of revolution; they were to visualize the region, plot the three-dimensional figure in a two-dimensional drawing, in their notebooks. Construct the three-dimensional figure in the software from well-defined functions. Here difficulties were detected in the students in the following steps: structuring, 
although the graph (model) constructed was correct, it did not offer them the possibility of identifying the algebraic expression(s) that represent the region, because it did not take into account the knowledge acquired previously. We identified that the transition between the graphic and algebraic register was quite difficult for them. We pointed out that in some cases the equations presented did not correspond to the figure drawn. We led them to reflect on this situation and to make adjustments. This aspect made mathematics difficult. Here in particular, difficulties were identified in calculating the volume, given that some students did not relate it to a solid of revolution, we believe because they have not developed the ability to coordinate results and intuitions in order to present them formally.

When presenting failures in the two steps mentioned in the previous paragraph, we identify that the interpretation also failed, manifested in difficulties to recognize and relate that the volume is finite but the surface area is infinite, this for the Torricelli trumpet, Figure 4 shows the production on paper of the student E20 who finds it difficult to understand the calculations made; in the lower part he writes: "why area and volume are different? I assumed that both diverged" this allows us to evidence that for him, the fact of thinking that the function tends to infinity, implicitly in his mind, the integral must be divergent, this reasoning is provoked by the graphic register given that the trumpet extends "infinitely". Here interest arose in the researchers to determine how many students realized this situation. We observed 16 out of 45, which allows us to infer that most of them show a lack of meaning of previous concepts and relate them to the graphic register; in terms of [14] this is an obstacle of linking compactness, because it seems that these students only conceive of a volume as finite if the figure is closed and bounded. A similar situation arose when working with other of the ten situations proposed, among them: a) calculate the work done to raise an object of mass $m$ to a defined height $h$; then determine the work required to propel it to an infinite distance from the Earth. b) Determine the force of gravity exerted by a uniform rod whose density is $\rho=4 \frac{\mathrm{Kg}}{\mathrm{m}}$ and which occupies the entire positive part of the $x$ axes $(x \geq 0)$, on a mass $m=1 \mathrm{Kg}$, located at the coordinate point $(-2 m, 0)$.
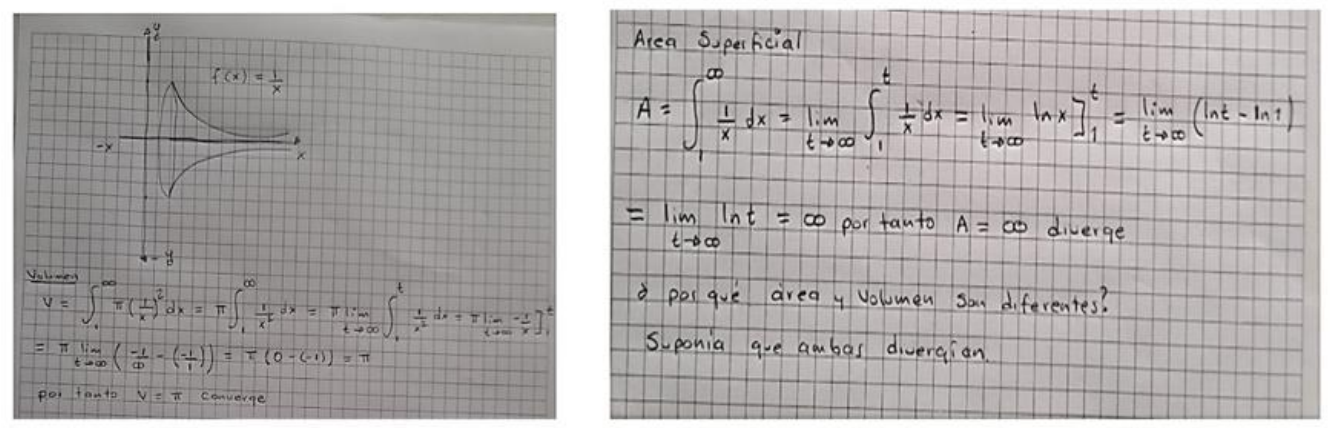

Figure 4. Student production E20.

The work carried out in this phase highlighted the creativity of the students, given that each subgroup, when faced with the construction of the proposed figure, had to imagine it decomposed into two-dimensional pieces. This activity allowed them to 
understand the importance of the continuity of a function, to identify domain, range, and to establish the difference between function and relation. As well as the transition between the graphic representation and its corresponding equation. In other words, to overcome the difficulties encountered in the steps: mathematics, resolution, interpretation.

Progressively we sought to integrate mathematics with other areas of knowledge, particularly physics, which allowed us to observe in the students, interest in mathematics in relation to its applicability; apprehension of mathematical concepts and development of skills of advanced mathematical thinking, sharing the position put forward by [9] (p. 69) "in higher education, mathematics is an essential part of the learning process, progressive matematization implies the need to abstract, define, analyse and formalize", and among the cognitive processes with a psychological component: "representing, conceptualizing, inducing and visualizing" (idem, p. 70).

\section{Conclusions}

The use of mathematical computer programmes offers students: clarity in the execution and hierarchy of operations, recognizing the graph of a function as a category that articulates modelling and technology. Here we share the position of [15-16] that the use of technological resources as a learning factor, where the electronic device (artefact) becomes an instrument for graphing, calculating and solving situations, makes it possible to implement the modelling-technology duo in the construction of mathematical knowledge in the classroom.

The proposed modelling process allowed students to go from using the educational software as a learning tool (instrumentalization) to using it as a learning tool (instrumentation) through interaction with it, improving their mathematical reasoning. In terms of [17], modelling as a didactic strategy effectively brings first semester students closer to the conceptual and algorithmic dimensions of calculus, allowing them to establish a connection between the concepts of Integral Calculus, the algorithms that characterize it, and which are present in their professional work. Among the weaknesses found that need further work is the commitment in the validation and exposition steps. Although the students try to make interpretations of the results, they are not used to explaining them, this situation generates insecurity in the work carried out and the belief, sometimes false, in the results of the software as always truthful and infallible, without considering that, if the data are recorded incorrectly, the software will produce inconsistencies such as those described in previous paragraphs. We therefore consider it necessary to recognize that the use of intuitive reasoning, both algebraic and graphical, is related to the level of understanding of the concepts involved, so that students with large conceptual gaps are unlikely to be able to use this type of reasoning.

We observed the widespread belief that the properties of a figure, in a number of dimensions, are maintained as this number increases or decreases; thus, a figure of infinite area should generate an infinite volume; possibly due to the absence of coordination between registers; what [18], call the presence of the obstacle of homogenizing dimensions. There was also evidence of a static conception of the process of limit as a simple algebraic 
operation, identified in the difficulties in conceptualizing the calculation of the area of a figure of infinite aspect.

Among the errors analysed, there is a lack of meaning when misinterpreting statements of a theorem or criterion and using it to solve a situation that is not related to the case, leading to incorrect answers. For example, thinking that a function that is symmetrical with respect to an axial axis, they evaluate it in an integration subinterval, then multiply it by 2 , thinking that they will obtain the value of the total integral, as if the axis of symmetry were the $Y$-axis of the plane. This confirms a lack of coordination between the graphic and algebraic registers, as well as a poor understanding of the definition of the definite integral. Not being clear about the criteria and their use reflects a lack of connections with the meanings attributed to the integral. Hence, we consider that a correct understanding of the concept of improper integral requires the student to visualize the calculation of areas as a dynamic process, which allows him to conceive the integral function and then calculate its limit, and not only to think of it as an area that must always be positive.

\subsection{Difficulties encountered in developing the proposal}

In one of the situations ${ }^{8}$, it was necessary to work on the area between curves, where the student had to pose the definite integral and solve it from the graph provided. In the algebraic resolution, they extrapolate the procedure using a piecewise function in different subintervals, thus showing an adequate handling of the instrumental procedures. However, in this situation we observe a limited development of conceptual thinking, given that the students are prevented from moving through the different semiotic representations, as they find it difficult to establish a connection between the results obtained with the algebraic and graphical registers, the inability to coherently identify the information provided by the problem and the lack of coherent coordination between the different registers used was evident. They solve the task correctly with the help of the software, but make mistakes on paper; the fact that the function is piecewise continuous seems to have a noticeable influence on their reasoning. Therefore, we consider that: to pose this type of situation requires the creativity of the teacher, since the evidence shows that not many of these activities are found in the textbooks proposed in the course bibliography.

There was evidence of a lack of meaning of fundamental tools for understanding concepts, such as: use of limits, notion of convergence, definition of definite integral, and some minimal knowledge of sequences and series (convergence criteria), without which it would be difficult to acquire a proper understanding of the concept of improper integral. This is a subject that remains open for further research. This ratifies the need for more research projects aimed at renewing the teaching-learning methodologies of basic sciences for university students, a subject that is little dealt with. 


\section{Notes}

1 Definite integral covering an unbounded area; where one or both limits of integration are infinite or when the integrand considers a function with a finite number of discontinuities in the interval in question.

2 The meaning of a mathematical object refers to the use given to it in the language game in which it participates. [18] indicates that the meaning (of a mathematical object) is linked to the problems in which it is involved and to the actions carried out to solve those problems.

3 It was previously pointed out to them that this type of twist in the function generates a solid of revolution.

4 Image available online at https://maskupnfm.wordpress.com/2011/11/10/cuerno-degabriel/

5 Solid of revolution generated by rotating around the $x$-axis the surface bounded by the curve $y=1 / x$ and the $x$-axis of the plane. Source: https://www.geogebra.org/m/th9kdb8c

6 For a better understanding of these difficulties, it is recommended to go deeper into the epistemological and cognitive aspects of these concepts.

7 This function evaluates the probability that a particular event occurs at some number in the interval $[0,1]$.

8 Build a tunnel that is $2.3 \mathrm{~km}$ long by $15 \mathrm{~m}$ wide. The shape of the tunnel is an arc whose equation is $y=25 \cos (\pi x / 50)$. They then had to calculate the area under the assumption that the tunnel was infinite in length

Author contributions: All authors have sufficiently contributed to the study, and agreed with the results and conclusions.

Funding: This work was total supported by own resources.

Declaration of interest: No potential conflict of interest was reported by the authors.

Acknowledgements: The authors thank the teachers and students who participated in this research and agreed to be interviewed and replied to the questionnaires.

Data Availability Statement: The data supporting this study will be made available by the corresponding author 1 upon reasonable request.

Conflicts of Interest: The authors declare no conflict of interest, as we worked as a team, sharing responsibilities equally throughout the research.

Institutional Review Board Statement: Not applicable

\section{References}

1. Blum,W. Mathematical modelling in mathematics education and instruction. In Breiteig, T., Huntley, I. and Kaiser-Messmer, G., (Eds.), Teaching and learning mathematics in context, Chichester, UK: Ellis Horwood. 1993. 3-14.

2. Muñoz-Ortega, G. Hacia un campo de prácticas sociales como fundamento para rediseñar el discurso escolar del Cálculo Integral. Revista Latinoamericana de Investigación en Matemática Educativa, 2010. 13(4), 283-302.

3. Scheja, M. \& Petterson, K. Transformation and contextualisation: Conceptualising students' conceptual understanding of threshold concepts in Calculus. Higher Education, 2010. 59(2), 221-241.

4. Alanís, J. \& Soto, A. La Integral de funciones de una variable: Enseñanza Actual. El Cálculo y su Enseñanza, 2012. 3(1), 1-6. 
5. Thompson, P., Byerley, C., \& Hatfield, N. (2013). A conceptual approach to Calculus made possible by technology. Computers in the Schools, 2013. (3), 124-147.

6. Mateus-Nieves, E. Análisis Didáctico a un Proceso de Instrucción del Método de Integración por Partes. Bolema, Rio Claro (SP), ISSN 1980-4415. 2016. 30 (55), 559-585. DOI: http://dx.doi.org/10.1590/1980-4415v30n55a13.

7. Mateus-Nieves, E. (2019). Extensiones al concepto de integral. Conference: Didactic Meeting of Calculus (infinitesimal). Bogotá, D. C. Project: Doctorado en Educación Matemática. DOI:10.13140/RG.2.2.34282.95689.[CrossRef]

8. Mateus-Nieves, E.; Hernandez, W. Significado Global de la Integral Articulando su Complejidad Epistémica. Revista Iberoamericana de Educación Matemática. UNION, ISSN: 1815-0640. Año XVI, 2020. 60, 196-211. [CrossRef]

9. Mateus-Nieves, E.; Rojas, C. Mathematical generalization since the articulation of advanced mathematical thinking and knot theory. Acta Scientiae, (Canoas). Volume 22 (3), pp. 65-81, Maio/Jun. 2020. ISSN: 2178-7727. DOI:10.17648/acta.scientiae.5567.

10. Mateus-Nieves, E. Una reflexión sobre las Integrales Impropias. Conference: Didactic Meeting of Calculus (infinitesimal). Bogotá, D.C. Project: Doctorado en Educación Matemática. DOI:10.13140/RG.2.2.20792.37123. 2020. [CrossRef]

11. Verillon, P.; Rabardel, P. Cognition and Artefacts: A contribution to the study of thought in relation to instrumented activity. European Journal of Psychology of Education, 1995. 10, 77-103.

12. Chevallard, Y. Analyse des pratiques enseignantes et didactique des mathématiques : l'approche anthropologique, en Actes de l'U. E. de la Rochelle: Analyse des pratiques enseignantes et didactique des mathématiques. 1998. 91-119.

13. Rabardel, P. Los hombres y las tecnologías: Visión cognitiva de los instrumentos contemporáneos (Trad. por M. Acosta). Universidad Industrial de Santander. 2011.

14. González-Martín, S. \& Camacho, M. The comprehension of the concept of improper integral in Mathematics students: representation and transference, en Giménez, J., Hahn, C. y Fitzsimons, G. (eds.). Proceedings of the 54th CIEAEM. 2002.

15. Trouche, L. Managing the complexity of human/machine interactions in computerized learning environments: guiding students command process through instrumental orchestrations. International Journal of Computers for Mathematical Learning, 2004. 9(3), 281-307.

16. Defouad, B. Etude de genèse instrumentale liées à l'utilisation d'une calculatrice symbolique en classe de première. Thèse de doctorat no publie. Université Paris7 France. 2000.

17. Blomhøj, M. Mathematical modelling-A theory for practice. International Perspectives on Learning and Teaching Mathematics, 2004. 1, 145-159.

18. Godino, J. Teoría de las funciones semióticas. Un enfoque ontológico-semiótico de la cognición e instrucción en matemática educativa. Recherches en Didactique des Mathématiques, 2002. 22 (3), 237-284. 